\title{
Impact of genital warts on health related quality of life in men and women in mainland China: a multicenter hospital-based cross-sectional study
}

\author{
Ju-Fang Shi ${ }^{1,2}$, Dian-Ju Kang ${ }^{1,3}$, Shu-Zhen Qi ${ }^{4}$, Hai-Yan Wu ${ }^{5}$, Yan-Chun Liư ${ }^{6}$, Li-Jun Sun ${ }^{7}$, Li Li $^{8}$, Ying Yang ${ }^{9}$, \\ Qing $\mathrm{Li}^{10}$, Xiang-Xian Feng ${ }^{11}$, Li-Qin Zhang ${ }^{12}$, Jie Li ${ }^{13}$, Xiao-Li $\mathrm{Li}^{14}$, Yun Yang ${ }^{15}$, Mayinuer Niyazi ${ }^{16}$, Ai-Di Xu ${ }^{17}$, \\ Jia-Hua Liu ${ }^{18}$, Qing Xiao ${ }^{19}$, Lian-Kun Li ${ }^{20}$, Xin-Zheng Wang ${ }^{21}$ and You-Lin Qiao ${ }^{1 *}$
}

\begin{abstract}
Background: Information on the health-related quality of life (HRQoL) of patients with genital warts (GW) in populations in mainland China is still limited. The aim of the study was to use a generic instrument to measure the impact of genital warts on HRQOL in men and women in this setting.

Methods: A multi-centre hospital-based cross-sectional study across 18 centers in China was conducted to interview patients using the European quality of life-5 dimension (EQ-5D) instrument; respondents' demographic and clinical data were also collected.

Results: A total of 1,358 GW patients ( 612 men, 746 women) were included in the analysis, with a mean age of $32.0 \pm 10.6$ years. $56.4 \%$ of the patients reported some problems in the dimension of Anxiety/Depression (highest), followed by Pain/Discomfort (24.7\%) and Mobility (3.5\%). The overall visual analogue scale (VAS) score of the study population was found to be $65.2 \pm 22.0$, and the EQ-5D index score was found to be $0.843 \pm 0.129$ using Japanese preference weights (the Chinese preference was unavailable yet). Patients with lower VAS means and EQ-5D index scores were more often female, living in urban area, and suffering multiple GW (all p values $<0.05$ ), but the values did not differ notably by age ( $p$ values $>0.05$ ).
\end{abstract}

Conclusions: The HRQoL of patients with GW was substantially lower, compared to a national representative general population in China (VAS $=\sim 80$ ); the findings of different subgroups are informative for future GW prevention and control efforts.

\section{Background}

Genital warts (GW) is one of the most common sexually transmitted diseases and about $90 \%$ of GW are caused by infection with human papillomavirus (HPV)-6 and HPV-11 [1]. The incidence of GW is increasing globally although some developed countries, such as US and Australia, have reported relatively low rates $(<0.3 \%)$ $[2,3]$. Information on the disease burden of GW in mainland China is still limited, but a relatively higher GW prevalence $(0.5 \%)$ was reported in general populations of other Asian regions (including India, Taiwan

\footnotetext{
* Correspondence: qiaoy@cicams.ac.cn

'Department of Cancer Epidemiology, Cancer Institute/Hospital, Chinese Academy of Medical Sciences, Peking Union Medical College, 17 South

Panjiayuan LN, PO Box 2258, Beijing 100021, China

Full list of author information is available at the end of the article
}

and Hong Kong) [1]. However, the variability in the GW prevalence among different data sources from other Asia Pacific countries might suggest that under-reporting potentially existed in the population surveillance systems [1]. In addition, a high post-treatment recurrence rate of GW has been reported (40\%) [4] and this characteristic of GW imposes a considerable sociopsychological burden (such as worries and concerns, negative emotional and sexual impact, shame or stigma, and worse interactions with partner and/or doctors $[5,6]$ ) and economic burden [7] on the patients.

It has been estimated that multivalent prophylactic HPV vaccine has the potential to protect up to $83 \%$ of genital warts or condylomata acuminate in mainland China [8]. In an era of HPV vaccination, a number of 
cost-effectiveness analyses (CEA) on multivalent HPV vaccines have been performed globally. Health related quality of life (HRQoL) contains self-reported measures of physical and psychological aspects, and an accurate impact analysis of genital warts on HRQoL was expected to contribute to the health economic evaluation on (HPV) vaccines protects against related HPV types. The extent of HRQoL of patients with GW has previously been quantified in different settings using generic instruments [6,9-11], including the European quality of life-5 dimension (EQ-5D) measure. EQ-5D is a standardized and commonly used instrument on HRQoL [12], and is also one of only a few measures recommended for use in cost-effectiveness analysis $[13,14]$. However, most of the quality of life studies using EQ-5D in China's populations were more focused on the general populations or patients with various chronic diseases [15-18], and assessment related to GW in this setting has not been performed.

The Cancer Foundation of China (CFC) and the Cancer Institute of Chinese Academy of Medical Sciences (CICAMS) jointly conducted a multi-centre hospitalbased epidemiological and economic study on genital warts across China. As part of this broad project, the current study's aim was to use the generic instrument EQ-5D to measure the impact of genital warts on health related quality of life in men and women in mainland China.

\section{Methods}

From July 2007 to July 2008, patients with genital warts were enrolled in 18 selected centers across seven geographic regions of China (Northeast, North, Northwest, Central, Southwest, South and East China). Generally, within each region, one province-level centre in a more developed area, and one county-level centre in a less developed area were selected. In addition, four additional study sites were selected from the North and East China regions in order to provide more information on more developed areas or metropolitan cities. Thus, a total of 11 higher level centers and 7 lower level centers eventually participated in the current survey (Figure 1).

A total of $100 \mathrm{GW}$ patients were initially required for each centre at a higher level and 50 patients at a lower level, and a convenience sampling method was applied within each centre. Patients who were currently diagnosed with genital warts, aged 18 years or older and provided informed consent (no participation fee applied) were eligible for this study regardless of their gender or whether they were an incident or recurrent case. Central institutional review board approval was obtained from the Cancer Foundation of China.

The EQ-5D instrument has several language versions and has been applied to a wide variety of populations

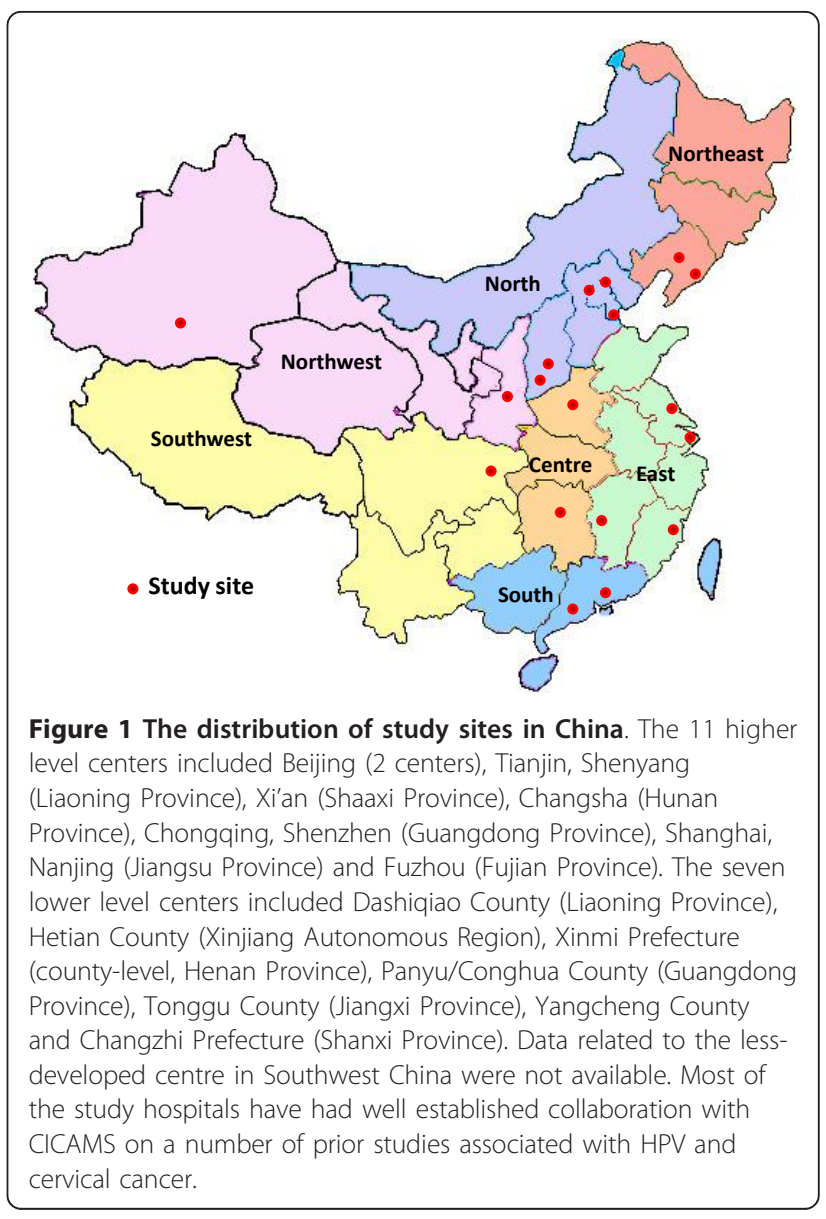

and diseases [12], and promising evidence for the measurement equivalence of EQ-5D's English and Chinese versions has been reported [19]. EQ-5D contains five dimensions measurement, including Mobility, Self-care, Activities, Pain/Discomfort and Anxiety/Depression [12]. Three options are listed for each dimension: whether the subject has no problem, a moderate problem or an extreme problem. Separately, EQ-5D instrument also contains a visual analogue scale (VAS) which allows the study subjects themselves to assess and self-record their health status from 0 (the worst) to 100 (the best) [12]. Using the EQ-5D instrument (Chinese version), a faceto-face interview was administrated by a project staff member, or alternatively a capable patient completed the questionnaire by him or herself in the presence of a trained interviewer who answered any questions the participants had about the questionnaire. In addition, further patient information including place of residence, education, income, marriage status, whether or not the patient is a smoker, sexual behavior and clinical characteristics were also collected. Information from patients who did not accept the invitation was not recorded in the study. 
Prior to the larger-scale survey, a pilot study was conducted in three sites (urban: Beijing; rural: Shanxi and Jiangxi); the initial study flowchart and questionnaire contents (excluding the EQ-5D questionnaire) were then modified and optimized based on the feedback from the pilot study. An investigators' meeting was then organized by CICAMS and CFC to provide data collection training and to introduce the study protocol and an operations manual to all the co-principle investigators and the main project members from 18 sites. The meeting participants were then required to provide training to other project team members (including nurses and physicians at the clinics) in local individual centers, based on the study operation manual. The operations manual contained questionnaire instructions, coding, quality control process, collaborators' responsibility, logistic instructions and other details in an operational level. CICAMS was responsible for all database building, logistical data checks (by interacting with local staff) and data analysis. Data collection and double entry and basic data checks were conducted in the 18 collaborative centers/hospitals.

The characteristics of the study population were described in general terms. Percentages of patients reporting any problems, age-specific means of EQ-5D VAS scores and their 95\% confidence intervals were analyzed (overall and by sex), using Chi-square test or one-way ANOVA approach. Because of the absence of a EQ-5D preference weight set for the Chinese population, we applied the preference weight sets from other populations (the UK, US and Japan) [20-22] to estimate the mean EQ-5D index score of this GW population in China. Detailed VAS means and EQ-5D index scores by population characteristics were calculated and a oneway ANOVA approach was applied to detect the statistical differences between subgroups. In addition, Pearson correlation among VAS means and EQ-5D index scores using the three preference weight sets were characterized using a univariate linear regression model. Since a very high association between weighted and nonweighted EQ scores has been reported [23], we then conducted a multivariate linear regression analysis to check the robustness of important variables, by inputting variables with statistical significance confirmed in the non-weighted analysis. For the ordinary unordered categorical variables with more than two subgroups, dummy variables were applied and the subgroup with lowest mean/score (which was southwest in the region category and recurrent GW in the clinical status category) was chosen as the reference subgroup. All analysis used two tailed tests.

\section{Results}

A total of 1,358 outpatients with genital warts were included in the analysis (612 men and 746 women), with a mean age of 32.0 years (SD: 10.6, range: 18-86). Of all study patients, $75.1 \%$ of them were enrolled from urban-based hospitals, $33.2 \%$ received college or higher education, $30.0 \%$ held health insurance, $22.2 \%$ were current frequent smokers, and their median monthly income was 1,400 Chinese Yuan ( 200 US\$). In addition, $69.3 \%$ of the study subjects were married or living with their partners, and $54.3 \%$ have had two or more lifetime sexual partners. During the survey, the patients were basically categorized into three clinical statuses, including the initial clinical visit for GW, the follow-up visit within an episode, and visits for recurrent GW, with the proportions in each status being $76.0 \%, 10.8 \%$ and $13.3 \%$, respectively. In all, $26.2 \%$ of the subjects were observed to have a single genital wart on their bodies, while the rest suffered multiple genital warts.

Table 1 illustrates the results of the five study dimensions. For the whole study population, the dimension of Anxiety/Depression had the highest rate of self-reported problems (56.4\%), followed by Pain/Discomfort (24.7\%), Mobility (3.5\%), Usual activities (2.8\%) and Self-care $(0.5 \%)$. Compared to the male patients, females tended to report more problems in three dimensions, including Anxiety/Depression (50.1\% vs. 61.5\%), Pain/Discomfort (20.6\% vs. $28.0 \%$ ), and Mobility (1.3\% vs. $5.2 \%$ ) (all $P$ values $<0.005)$. The overall VAS mean among all cases was 65.2 (SD: 22.0), and men had a higher mean score than women $(69.0$ vs. $62.1, P<0.001)$. To make comparisons for all of the reported groups, relevant findings from a national population-based survey in China [15] were also added to Table 1 as references. After applying the preference weights of the Japanese, UK and US populations, the overall EQ-5D index scores of the current analysis were $0.843,0.826$ and 0.859 , respectively; and similarly, men scored higher than women under every one of the three preference weight sets (all $P$ values $<0.001$ ) (Table 2).

A range of strengths of correlation between results from different analyses were characterized. On one hand, relatively low Pearson correlations $\mathrm{R}$ between EQ VAS mean and EQ-5D index scores were observed (0.371, 0.423, 0.412 using Japanese, UK and US preference weights respectively, all $p$ values $<0.001)$. On the other hand, unsurprisingly, a slightly higher agreement (0.984, $p<0.001)$ was observed between EQ-5D index scores of UK and US preference weights compared to those of the other two pairs (Japan \& UK: 0.817, Japan \& US: 0.860 , all $p$ values $<0.001)$; this situation is potentially due to the cultural dissimilarity between Western and Asian populations. In addition to this, compared to the EQ-5D results for populations in the UK and US, the reported percentages of subjects' problems by each of the EQ-5D's five dimensions among the Japanese general population were closer to those of 
Table 1 Percentages of subjects self-reported problems and means of visual analogue scale (VAS), total and by sex

\begin{tabular}{|c|c|c|c|c|c|c|c|c|c|}
\hline \multirow[t]{3}{*}{ EQ-5D dimension } & \multicolumn{6}{|c|}{ The current study } & \multicolumn{3}{|c|}{$\begin{array}{l}\text { Findings from a national population-based survey in China, } 2008 \\
{[15]}\end{array}$} \\
\hline & \multicolumn{2}{|r|}{ Male } & \multicolumn{2}{|r|}{ Female } & \multicolumn{2}{|r|}{ Total } & \multirow{2}{*}{$\begin{array}{c}\text { Male } \\
\%(\mathrm{~N}=58,163)\end{array}$} & \multirow{2}{*}{$\begin{array}{c}\text { Female } \\
\%(\mathrm{~N}=62,540)\end{array}$} & \multirow{2}{*}{$\begin{array}{c}\text { Total }^{*} \\
\%(\mathrm{~N}=120,703)\end{array}$} \\
\hline & No. & $\%$ & No. & $\%$ & No. & $\%$ & & & \\
\hline \multicolumn{10}{|l|}{ Mobility } \\
\hline No & 604 & 98.7 & 707 & 94.8 & 1,311 & 96.5 & 95.7 & 94.6 & 95.1 \\
\hline Moderate & 8 & 1.3 & 38 & 5.1 & 46 & 3.4 & 4.0 & 5.0 & 4.5 \\
\hline Extreme & 0 & 0.0 & 1 & 0.1 & 1 & 0.1 & 0.3 & 0.4 & 0.4 \\
\hline \multicolumn{10}{|l|}{ Self-care } \\
\hline No & 610 & 99.7 & 741 & 99.3 & 1,351 & 99.5 & 97.3 & 96.6 & 96.9 \\
\hline Moderate & 2 & 0.3 & 5 & 0.7 & 7 & 0.5 & 2.3 & 3.0 & 2.7 \\
\hline Extreme & 0 & 0.0 & 0 & 0.0 & 0 & 0.0 & 0.4 & 0.4 & 0.4 \\
\hline \multicolumn{10}{|l|}{ Usual activities } \\
\hline No & 596 & 97.4 & 723 & 96.9 & 1,319 & 97.1 & 96.0 & 94.9 & 95.4 \\
\hline Moderate & 16 & 2.6 & 21 & 2.8 & 37 & 2.7 & 3.3 & 4.3 & 3.8 \\
\hline Extreme & 0 & 0.0 & 2 & 0.3 & 2 & 0.1 & 0.7 & 0.8 & 0.8 \\
\hline \multicolumn{10}{|l|}{ Pain/Discomfort } \\
\hline No & 486 & 79.4 & 537 & 72.0 & 1,023 & 75.3 & 92.8 & 89.5 & 91.1 \\
\hline Moderate & 122 & 19.9 & 205 & 27.5 & 327 & 24.1 & 6.9 & 10.0 & 8.5 \\
\hline Extreme & 4 & 0.7 & 4 & 0.5 & 8 & 0.6 & 0.3 & 0.5 & 0.4 \\
\hline \multicolumn{10}{|l|}{ Anxiety/Depression } \\
\hline No & 305 & 49.8 & 287 & 38.5 & 592 & 43.6 & 94.8 & 92.9 & 93.8 \\
\hline Moderate & 259 & 42.3 & 362 & 48.5 & 621 & 45.7 & 4.9 & 6.7 & 5.8 \\
\hline Extreme & 48 & 7.8 & 97 & 13.0 & 145 & 10.7 & 0.3 & 0.4 & 0.4 \\
\hline \multicolumn{10}{|l|}{ VAS score } \\
\hline & 612 & 69.0 (mean) & 746 & 62.1 (mean) & 1,358 & 65.2 (mean) & 80.9 (mean) & 79.4 (mean) & 80.1 (mean) \\
\hline
\end{tabular}

Chi-square tests were performed, female patients tended to report more problems in three dimensions, including Anxiety/Depression, Pain/Discomfort and Mobility, when comparing to male patients (all $P$ values $<0.005$ ).

* The percentages of "Total" group were estimated based on the reported findings for males and females with considering each group's sample size.

the general population in China [15,17]. For this reason, only the preference weight set of the Japanese population was applied when we calculated further characteristic-specific EQ-5D index scores in this analysis.

Table 3 presents further detailed mean VAS and EQ-5D index scores for different subgroups. Briefly, female patients, living in urban areas, residing in the Southwest region, smoking less frequently and with multiple clinically-confirmed GW were statistically associated with

Table 2 The EQ-5D index score using different preference weights, total and by sex

\begin{tabular}{llll}
\hline \hline Preference weight set & Male & Female & Total
\end{tabular}
applied

\begin{tabular}{lcccccc}
\cline { 2 - 7 } & Mean & SD & Mean & SD & Mean & SD \\
\hline Japan & 0.864 & 0.13 & 0.827 & 0.126 & 0.843 & 0.129 \\
\hline UK & 0.856 & 0.185 & 0.802 & 0.21 & 0.826 & 0.201 \\
\hline US & 0.882 & 0.137 & 0.84 & 0.15 & 0.859 & 0.145
\end{tabular}

One-way ANOVA approach was applied to compare scores between men and women

(all $P$ values $<0.001$ ) lower VAS and EQ-5D index scores (one-way ANOVA results, all $p$ values $<0.05$ ); and a lower monthly income and the situation of recurrent GW probably contributed to a worse HRQoL of GW patients $(P<0.05$ in the EQ-5D index score calculation, but $P>0.05$ in the VAS analysis). Significant differences were not detected among subgroups by age (Figure 2), marital status, education, insurance or the number of lifetime sexual partners (all $P$ values $>0.05$ ).

In the multivariate linear regression analysis, similar results were observed (Table 4); when using the VAS mean as the dependent variable, the variables of gender, rural or urban, residence in the North or Central regions of China, and single/multiple GW were retained in the final model (all $P$ values $<0.01$, adjusted $\mathrm{R}$ square $=$ 0.065). When placing EQ-5D index score as the dependent variable, more factors entered the model, including initially occurred GW, monthly income, region of East, and region of Northeast (all $P$ values $<0.001$, adjusted R square $=0.117$ ) (Table 4). The factors of smoking and monthly income were not statistically associated with the HRQoL of GW patients in the multivariate analysis. 
Table 3 Mean visual analogue scale (VAS) and EQ-5D index score, by population characteristics*

\begin{tabular}{|c|c|c|c|c|c|c|c|c|}
\hline \multirow[t]{2}{*}{ Variable } & \multirow[t]{2}{*}{ No. } & \multirow[t]{2}{*}{$\%$} & \multicolumn{3}{|c|}{ VAS } & \multicolumn{3}{|c|}{ EQ-5D index score ${ }^{* *}$} \\
\hline & & & Mean & SD & Sig. ${ }^{\prime}$ & Mean & SD & Sig. ${ }^{\#}$ \\
\hline \multicolumn{9}{|l|}{ Sex } \\
\hline Male & 612 & 45.1 & 69.0 & 21.3 & $<0.001$ & 0.864 & 0.130 & $<0.001$ \\
\hline Female & 746 & 54.9 & 62.1 & 22.1 & & 0.827 & 0.126 & \\
\hline \multicolumn{9}{|l|}{ Region } \\
\hline North & 372 & 27.4 & 66.6 & 25.0 & $<0.001$ & 0.866 & 0.135 & $<0.001$ \\
\hline Northeast & 128 & 9.4 & 64.0 & 24.7 & & 0.897 & 0.125 & \\
\hline Northwest & 151 & 11.1 & 62.2 & 20.6 & & 0.790 & 0.121 & \\
\hline Central & 133 & 9.8 & 72.3 & 12.8 & & 0.871 & 0.120 & \\
\hline Southwest & 100 & 7.4 & 57.3 & 22.2 & & 0.790 & 0.111 & \\
\hline South & 135 & 9.9 & 66.1 & 18.6 & & 0.823 & 0.134 & \\
\hline East & 339 & 25.0 & 64.5 & 21.2 & & 0.835 & 0.120 & \\
\hline \multicolumn{9}{|l|}{ Setting } \\
\hline Urban & 1,020 & 75.1 & 63.2 & 22.4 & $<0.001$ & 0.837 & 0.126 & $<0.005$ \\
\hline Rural & 338 & 24.9 & 71.0 & 19.6 & & 0.862 & 0.137 & \\
\hline \multicolumn{9}{|l|}{ Be married / living together } \\
\hline Yes & 941 & 69.3 & 65.0 & 22.2 & $>0.05$ & 0.844 & 0.130 & $>0.05$ \\
\hline No & 417 & 30.7 & 65.5 & 21.6 & & 0.843 & 0.128 & \\
\hline \multicolumn{9}{|l|}{ Education } \\
\hline Middle school or lower & 408 & 30.0 & 66.3 & 20.7 & $>0.05$ & 0.842 & 0.132 & $>0.05$ \\
\hline High school & 499 & 36.7 & 66.1 & 22.0 & & 0.843 & 0.130 & \\
\hline College or above & 451 & 33.2 & 63.2 & 23.1 & & 0.845 & 0.127 & \\
\hline \multicolumn{9}{|l|}{ Monthly income (Chinese Yuan) * } \\
\hline$<1000$ & 364 & 26.8 & 63.5 & 22.4 & $>0.05$ & 0.827 & 0.133 & $<0.005$ \\
\hline $1000 \sim$ & 422 & 31.1 & 66.8 & 21.3 & & 0.838 & 0.124 & \\
\hline $2000 \sim$ & 223 & 16.4 & 65.8 & 22.8 & & 0.852 & 0.131 & \\
\hline $3000 \sim$ & 347 & 25.6 & 64.5 & 22.0 & & 0.860 & 0.128 & \\
\hline \multicolumn{9}{|l|}{ Any insurance coverage* } \\
\hline Yes & 432 & 31.8 & 65.8 & 21.8 & $>0.05$ & 0.842 & 0.129 & $>0.05$ \\
\hline No & 925 & 68.1 & 65.0 & 22.0 & & 0.844 & 0.131 & \\
\hline \multicolumn{9}{|l|}{ Frequently smoking } \\
\hline Yes & 301 & 22.2 & 68.2 & 21.2 & $<0.01$ & 0.860 & 0.131 & $<0.05$ \\
\hline No & 1,057 & 77.8 & 64.3 & 22.2 & & 0.839 & 0.129 & \\
\hline \multicolumn{9}{|l|}{ Number of lifetime sexual partners* } \\
\hline 1 & 612 & 45.1 & 63.8 & 22.7 & $>0.05$ & 0.852 & 0.132 & $>0.05$ \\
\hline 2 & 318 & 23.4 & 65.3 & 21.9 & & 0.839 & 0.128 & \\
\hline 3 or more & 421 & 31.0 & 67.0 & 21.0 & & 0.834 & 0.126 & \\
\hline \multicolumn{9}{|l|}{ Clinical status } \\
\hline First clinical visit for the initially occurred GW & 1,032 & 76.0 & 65.7 & 21.8 & $>0.05$ & 0.853 & 0.129 & $<0.001$ \\
\hline Follow-up visit for the initially occurred GW & 146 & 10.8 & 64.1 & 24.4 & & 0.822 & 0.126 & \\
\hline Recurrent GW & 180 & 13.3 & 63.9 & 21.0 & & 0.804 & 0.124 & \\
\hline \multicolumn{9}{|l|}{ Single or multiple genital warts } \\
\hline Single & 356 & 26.2 & 68.0 & 21.2 & $<0.01$ & 0.869 & 0.127 & $<0.001$ \\
\hline Multiple & 1,002 & 73.8 & 64.2 & 22.2 & & 0.835 & 0.129 & \\
\hline
\end{tabular}

* Some data missing; ** Japanese preference weights applied; " One-way ANOVA analysis.

\section{Discussion}

The current work is a unique large-scale multi-centre study focusing on the HRQoL of male and female patients with GW using a generic and internationally comparable instrument, the EQ-5D. We found that anxiety and depression posed a major problem for $\mathrm{GW}$ patients, and the measurement of VAS suggested that the HRQoL of GW patients was substantially lowered. The current analysis using different preference weights provided a range of utility estimates (overall and 


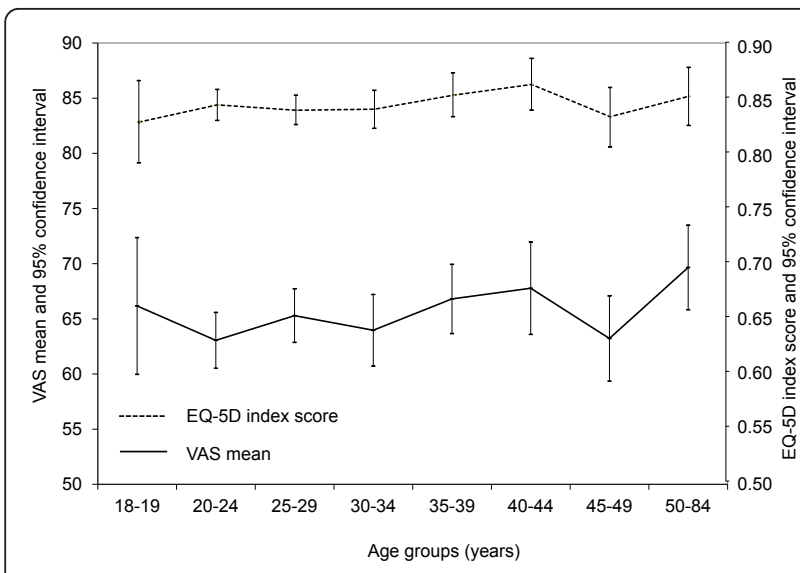

Figure 2 Age-specific means of visual analogue scale (VAS) and EQ-5D index score*. One-way ANOVA analyses were performed ( $P$ $>0.05) ; *$ Japanese preference weights applied.

characteristic-specific) for future detailed QALYrelated cost-effectiveness evaluations. This study also determined the characteristics of patents with relatively lower quality of life, including being female, living in urban areas and suffering multiple GWs, which will be informative for future GW prevention and control efforts.
The research group has published a parallel work conducted as another part of the whole GW project [5]. Both the studies explored the quality of life issue but from different perspectives and each study had its own emphasis. For example, firstly, the current study focused on both males and females, while the previous work mainly assessed female populations. Secondly, the current study was very focused on GW patients only, but the study by Wang et al included women who have a spectrum of HPV-related health statuses or laboratory diagnosis, including normal/abnormal pap smear, cervical precancer, and HPV $+/$ - after abnormal pap, and patients with GW were only one sub-group in the broad analysis [5]. Thirdly, the previous work used a newlydeveloped HPV-specific instrument, the HPV impact profile (HIP); it is more sensitive and can recognize some slight differences among the targeted HPV-related subgroup women, but it is more difficult to directly compare the findings of the HIP study and other quality of life studies using different instruments. Differently, the EQ-5D, used by the current analysis, is an easy-tocompare HRQoL instrument and commonly-used world widely [12]. Finally, compared to the HIP score outcomes, the EQ-5D index score outcomes reported by the current study were relatively more convertible when

Table 4 Mean VAS and EQ-5D index score, by population characteristics, using a multivariate linear regression approach

\begin{tabular}{|c|c|c|c|c|c|c|}
\hline \multirow[t]{2}{*}{ Variable } & \multirow[t]{2}{*}{ Variable definition } & \multicolumn{2}{|c|}{$\begin{array}{l}\text { Unstandardized } \\
\text { Coefficients }\end{array}$} & \multirow{2}{*}{$\begin{array}{c}\text { Standardized Coefficients } \\
\text { Beta }\end{array}$} & \multirow[t]{2}{*}{$\mathrm{t}$} & \multirow[t]{2}{*}{ Sig. } \\
\hline & & B & SD & & & \\
\hline \multicolumn{7}{|l|}{ VAS mean } \\
\hline Constant & & 63.721 & 1.455 & & 43.795 & 0.000 \\
\hline Sex & Woman $=0 ;$ Man $=1$ & 7.451 & 1.176 & 0.168 & 6.335 & 0.000 \\
\hline Setting & Rural = $0 ;$ Urban $=1$ & -6.459 & 1.361 & -0.127 & -4.747 & 0.000 \\
\hline Single/Multiple GW & Multiple GW = 0; Single GW = 1 & 4.138 & 1.334 & 0.082 & 3.101 & 0.002 \\
\hline Region-Central & Southwest $=0 ;$ Central $=1$ & 8.653 & 2.042 & 0.117 & 4.237 & 0.000 \\
\hline Region-North & Southwest $=0$; North $=1$ & 3.655 & 1.341 & 0.074 & 2.725 & 0.007 \\
\hline Region-East & Southwest $=0$; East $=1$ & 0.032 & 0.010 & 0.108 & 3.145 & 0.002 \\
\hline \multicolumn{7}{|l|}{ EQ-5D index score* } \\
\hline Constant & & 0.748 & 0.012 & & 61.166 & 0.000 \\
\hline Sex & Woman = 0; Man = 1 & 0.035 & 0.007 & 0.134 & 5.140 & 0.000 \\
\hline Setting & Rural $=0 ;$ Urban $=1$ & -0.032 & 0.008 & -0.106 & -3.933 & 0.000 \\
\hline Single/Multiple GW & Multiple GW = 0; Single GW = 1 & 0.029 & 0.008 & 0.098 & 3.749 & 0.000 \\
\hline Region-Central & Southwest $=0$; Central $=1$ & 0.067 & 0.013 & 0.153 & 5.332 & 0.000 \\
\hline Region-North & Southwest $=0 ;$ North $=1$ & 0.069 & 0.009 & 0.238 & 7.813 & 0.000 \\
\hline Region-East & Southwest $=0$; East $=1$ & 0.038 & 0.009 & 0.127 & 4.188 & 0.000 \\
\hline Region-Northeast & Southwest $=0$; Northeast $=1$ & 0.087 & 0.013 & 0.196 & 6.905 & 0.000 \\
\hline Clinical status- Recurrent GW & Recurrent GW = 0; Initially occurred GW = 1 & 0.031 & 0.008 & 0.103 & 3.968 & 0.000 \\
\hline Monthly income (Chinese Yuan) & $<1000=1 ; 1000 \sim=2 ; 2000 \sim=3 ; 3000 \sim=4$ & 0.012 & 0.003 & 0.107 & 4.040 & 0.000 \\
\hline
\end{tabular}

\footnotetext{
* Japanese preference weights applied.
} 
taking qualifying quality-adjusted life year (QALY) saved as the study outcome.

The current analysis found that more than half of the GW patients in this survey were suffering anxiety and depression. This is a dramatically high proportion, when compared to the value of $<7 \%$ of the sampled general population (aged 15-49 years, by 5 -year age group) across mainland China, as a part of the Chinese National Health Services Survey in $2008(\mathrm{~N}=120,703)$ [15]; and the proportion is also higher than that of some other countries $(\sim<30 \%$, pooled in a previous study by Wang et al) [17] and a group of GW patients in the UK (24\%) [9]. This situation is due mainly to the relatively conservative culture and attitude to sex in China; being diagnosed with sexually transmitted diseases such as GW could be regarded as a big humiliation for patients and they usually would not let other people know and would not receive support even from their families. As expected, we also found that a high proportion of study cases were feeling pain and discomfort $(24.7 \%)$ when compared to the Chinese general population ( $<10 \%$, in patients aged $15-49$ years) [15]. Providing questionnaire interview to part of the patients after their treatment could increase the feeling of pain, but the current study did not distinguish the ordering of treatment and interview. However, discomfort is commonly feeling in GW patients, which could explain some of this detriment. In contrast to the dimension of Anxiety/Depression, the dimensions of Mobility, Selfcare and Usual Activities in this GW population are generally less impacted when compared to local and international general populations [15,17]; this situation is due partly to the reality that most of the studied GW patients $(\sim 93 \%)$ were younger than 50 years old, and were able to move, to take care of themselves, and to complete their usual activities with no difficulty. Also, the situation of lower rates of any problems in the three dimensions mentioned above is consistent with the findings of a UK GW study [9].

A large-scale survey included EQ-5D instrument was previously conducted based on a national representative sample in China in 2008, where the average VAS were found to be 80.9 for male and 79.4 for female [15]. Since HRQoL scores are very age-dependent but the subjects of current study mainly aged 15-49 years, we restricted the comparison to narrower age groups (1549 years, by 5 -year age group); it was noted that the national survey reported relatively higher values of VAS score (81.4-89.8 for male, 79.2-89.6 for female). The finding that the mean VAS scores in the current study are lower than those of Chinese general population (65.2 versus $\sim 80[15]$ ) suggests that the HRQoL of patients with GW is notably lower than that of the general population. When compared to prior GW studies using EQ-5D in other countries, the mean VAS scores of the current Chinese GW cohort are lower than the estimate of an Australian study $(68.9, \mathrm{~N}=40)$ and of a UK study $(72, \mathrm{~N}=81)[6,9]$, but somewhat closer to the estimate of a Canadian study $(65.1, \mathrm{~N}=39)$ [11]. The observed differences between these GW cohorts in these settings might be explained by the differences in the VAS scores of the general populations behind them, which are 82.5, 78.7 and 80.1 for the UK, Canada and China, respectively. Based on results from other published works $[9,15,17]$, it is not surprising to see that female GW patients had lower VAS scores than male patients. Although a VAS score declining with age was observed in a general population-based survey [15], our age curve of VAS scores shows a flat pattern, which is consistent with a prior UK GW study [9]. A potential reason for this inconsistency is that the majority of the GW patients were sexually active, and they might have a lower probability of susceptibility to a range of ageing diseases or situations.

Due to the absence of an EQ-5D preference weight set in the Chinese population, three other populations' preference sets were applied to estimate the EQ-5D index scores in the current analysis. Our findings suggest that scores based on the Japanese preference weights could be regarded as the baseline utility values for future costeffectiveness evaluations, whilst the results based on the UK and US populations could provide a plausible range of utilities estimates for sensitivity analysis. When comparing our EQ-5D index scores to other international GW studies, a wide difference was noted but all our EQ-5D index scores using varied populations preference weights $(0.826,0.843$ and 0.859$)$ are within the range $(0.76-0.91)$ of the available data from other populations [9-11]; the lowest utility value (0.76) of GW patients was from a Canadian study, and the highest value (0.91) was reported by Myers et al (conference abstract, details unavailable) which has been cited by a number of more recent cost-effectiveness evaluations of quadrivalent HPV vaccine [24-27]. Our EQ-5D index scores analysis also further supports the hypothesis that female patients suffered a larger decrease in quality of life than males and the scores did not differ notably among age groups, as we previously discussed in the VAS scores analysis.

In addition to the variable of gender, other characteristics could also potentially affect the HRQoL of GW patients. Our finding that the urban patients suffered a heavier physiological burden than rural patients could be explained by the urban residents' higher stress from job and mortgage payments and living a faster-paced life. This finding is identical with the results of another HRQoL analysis of HPV-related lesions (including GW) using a HPV-sensitive instrument [5]. It is not surprising to observe that subjects in Southwest China had the 
lowest score, because a prior survey $(\mathrm{N}=2,830)$ has reported that the general population in Guizhou Province in the Southwest region of China had a relatively low HRQoL (VAS: $\sim 68-\sim 80$ in residents aged 15-49 years) [18]. It is also understandable that more patients with multiple genital warts reported lower scores than patients with only single GW. Although most of the differences detected between subgroups were marginally less than the mean of clinically important differences in EQ-5D (0.074) [28], they were still within the range (0.011-0.140) [28], and could potentially generate a relatively significant impact on mass public health intervention programs. As for other variables marginally associated with the HRQoL of GW patients, including initial or recurrent GW, smoking, patient's monthly income and education level, they are beyond the scope of the current discussion, and more research needs to be done.

This analysis has some limitations. Firstly, selection biases could occur in this study, due to the convenient sampling approach we used; we also failed to collect information of non-attended patients and thus could not assess the differences in characteristics between the study participants and those who declined to participate. Secondly, use of the generic EQ-5D instrument, which is not sensitive to HPV-related diseases, potentially underestimates the negative impact from GW and a ceiling effect could occur. Thirdly, the current study is a questionnaire interview-based survey which is usually sensitive to the capacities of the interviewers and quality control, and some of the clinical physicians who administrated the interview had not directly received training provided by CICAMS, and quality-control could potentially vary among study centers to some extent. Another limitation of the study is that we did not have Chinese population specific preference weights and thus could not calculate the EQ-5D index scores accurately. Furthermore, combined with further data related to average duration of a clinical episode and the frequency of recurrence of $\mathrm{GW}$, the detailed utilities findings from the current study would be informative for future costeffectiveness evaluations related to quality-adjusted life years saved by new interventions against GW.

\section{Conclusions}

The HRQoL of patients with GW was substantially lowered when compared to the general population in mainland China, and the quality of life was even worse among some sub-groups. It is vital to strengthen the public genital warts prevention program. In the era of HPV vaccination, although prophylactic HPV vaccines are not yet licensed in China, introducing a prophylactic vaccine protecting against HPV-6 and - 11 would be feasible. Regarding the psychological effect of such an intervention on GW patients, effectively reducing patients' anxiety and depression can have a remarkably positive impact on the overall quality of life of GW patients. In addition, particular attention should probably be given to female patients and patients from urban areas and who are suffering worse clinical situations in clinical practice.

\section{Abbreviations}

CFC: Cancer Foundation of China; CICAMS: Cancer Institute of Chinese Academy of Medical Sciences; EQ-5D: European quality of life-5 dimension; HRQoL: Health-related quality of life; GW: Genital warts; HPV: Human Papillomavirus; VAS: Visual analogue scale.

\section{Acknowledgements}

We wish to thank all members of the Chinese genital warts study group from Cancer Institute of Chinese Academy of Medical Sciences, Cancer Foundation of China and the 18 collaborative hospitals/universities. We also thank all the subjects who participated in this study. Dr. Karen Canfell and Mr. Robert Walker (Cancer Council NSW, Australia) are gratefully

acknowledged for comments and reviewing this manuscript. The study was unconditionally funded by MSD China.

\section{Author details}

'Department of Cancer Epidemiology, Cancer Institute/Hospital, Chinese Academy of Medical Sciences, Peking Union Medical College, 17 South Panjiayuan LN, PO Box 2258, Beijing 100021, China. ${ }^{2}$ Cancer Epidemiology Research Unit, Cancer Council NSW, New South Wales, Australia. ${ }^{3}$ Sichuan Center for Disease Control and Prevention, Chengdu, China. ${ }^{4}$ Institute of Dermatology, Chinese Academy of Medical Sciences, Peking Union Medical College, Nanjing, China. ${ }^{5} 202$ Hospital of Chinese PLA, Shenyang, China. ${ }^{6}$ Beijing Ditan Hospital, Beijing, China. 'Beijing You'an Hospital, Beijing, China. ${ }^{8}$ Daping Hospital, Third Military Medical University of Chinese PLA, Chongqing, China. ${ }^{9}$ Tangdu Hospital, Fourth Military Medical University of Chinese PLA, Xi'an, China. ${ }^{10}$ Shenzhen Women and Children Hospital, Shenzhen, China. ${ }^{11}$ Changzhi Medical College, Changzhi, China. ${ }^{12}$ General Hospital of Tianjin Medical University, Tianjin, China. ${ }^{13}$ Hunan Provincial People's Hospital, Changsha, China. ${ }^{14}$ Xinmi Women and Children Hospital, Xinmi, China. ${ }^{15}$ Medical School of Yichun University, Yichun, China. ${ }^{16}$ People's Hospital of Xinjiang Uygur Autonomous Region, Urumchi, China. ${ }^{17}$ Hongkou District Bureau of Health, Shanghai, China. ${ }^{18}$ Fujian Provincial Hospital, Fuzhou, China. ${ }^{19}$ Women and Children Hospital of Guangzhou City, Guangzhou, China. ${ }^{20}$ Liaoning Cancer Hospital, Shenyang, China. ${ }^{21}$ The 2nd People's Hospital of Yangcheng County, Shanxi, China.

\section{Authors' contributions}

YLQ was the Project Investigator and supervised the conduct of the study. YLQ and JFS contributed to the study design. DJK, SZQ, HYW, YCL, LS, LL, $Y Y, Q L, X X F, L Q Z, J L, X L L, Y Y, M N, A D X, J H L, X Q, L K L$ and $X Z W$ collected the field data. JFS performed all the data analyses with input and comments from YLQ. JFS wrote the first draft of the paper with input and comments from all other authors. All authors read and approved the final manuscript.

\section{Competing interests}

The authors declare that they have no competing interests.

Received: 20 October 2011 Accepted: 2 March 2012

Published: 2 March 2012

\section{References}

1. Parkin DM, Louie KS, Clifford G: Burden and trends of type-specific human papillomavirus infections and related diseases in the Asia Pacific region. Vaccine 2008, 26(Suppl 12):M1-16.

2. Pirotta M, Stein AN, Conway EL, Harrison C, Britt H, Garland S: Genital warts incidence and healthcare resource utilisation in Australia. Sex Transm Infect 2010, 86:181-186.

3. Koshiol JE, Laurent SA, Pimenta JM: Rate and predictors of new genital warts claims and genital warts-related healthcare utilization among 
privately insured patients in the United States. Sex Transm Dis 2004 31:748-752.

4. O'Mahony C: Genital warts: current and future management options. Am J Clin Dermatol 2005, 6:239-243.

5. Wang SM, Shi JF, Kang DJ, Song P, Qiao YL: Chinese HPV Study Group: Impact of Human Papillomavirus Related Lesions on Quality of Life: A Multicenter Hospital-Based Study of Women in Mainland China. Int $J$ Gynecol Cancer 2011, 21:182-188.

6. Pirotta M, Ung L, Stein A, Conway EL, Mast TC, Fairley CK, et al: The psychosocial burden of human papillomavirus related disease and screening interventions. Sex Transm Infect 2009, 85:508-513.

7. Wang D, Shi JF, Li L, Yan SW, Sun LJ, Li CQ, et al: Measuring direct costs of treating genital warts in China [abstract]. The 4th Asian Pacific Organization for Cancer Prevention General Assembly Conference, Beijing 2008.

8. Wang H, Qiao YL: Human papillomavirus type-distribution in condylomata acuminata of mainland China: a meta-analysis. Int J STD AIDS 2008, 19:680-684.

9. Woodhall S, Ramsey T, Cai C, Crouch S, Jit M, Birks Y, et al: Estimation of the impact of genital warts on health-related quality of life. Sex Transm Infect 2008, 84:161-166.

10. Myers E, Green S, Lipkus I: Patient preferences for health states related to HPV infection: visual analogue scales vs. time trade-off elicitation [abstract]. The 21st International Papillomavirus Conference, Mexico City, Mexico 2004.

11. Marra C, Ogilvie G, Gastonguay L, Colley L, Taylor D, Marra F: Patients with genital warts have a decreased quality of life. Sex Transm Dis 2009, 36:258-260.

12. EQ-5D Home page. 2011 [http://www.eurogol.org/]

13. Rabin R, de CF: EQ-5D: a measure of health status from the EuroQol Group. Ann Med 2001, 33:337-343.

14. Coons SJ, Rao S, Keininger DL, Hays RD: A comparative review of generic quality-of-life instruments. Pharmacoeconomics 2000, 17:13-35.

15. Sun $S$, Chen J, Johannesson M, Kind P, Xu L, Zhang Y, et al: Population health status in China: EQ-5D results, by age, sex and socio-economic status, from the National Health Services Survey 2008. Qual Life Res 2010, 20:309-320.

16. Li MH, Luo N: An introduction to application of EQ-5D Chinese version. China Journal of Pharmaceutical Ecomomics 2009, 1:49-57.

17. Wang H, Kindig DA, Mullahy J: Variation in Chinese population health related quality of life: results from a EuroQol study in Beijing, China. Qual Life Res 2005, 14:119-132.

18. Zhang LC, Wang H, Wang LS, Xiao QL: Health Related Quality of Life in West Rural Population. Chinese Health Economics 2005, 24:8-11.

19. Luo N, Chew LH, Fong KY, Koh DR, Ng SC, Yoon KH, et al: Do English and Chinese EQ-5D versions demonstrate measurement equivalence? An exploratory study Health Qual Life Outcomes 2003, 1:7.

20. Tsuchiya A, Ikeda S, Ikegami N, Nishimura S, Sakai I, Fukuda T, et al: Estimating an EQ-5D population value set: the case of Japan. Health Econ 2002, 11:341-353.

21. Dolan P: Modeling wfiuations for EuroQol health states. Med Care 1997, 35:1095-1108.

22. Shaw JW, Johnson JA, Coons SJ: US valuation of the EQ-5D health states: development and testing of the D1 valuation model. Med Care 2005, 43:203-220.

23. Prieto L, Sacristan JA: What is the value of social values? The uselessness of assessing health-related quality of life through preference measures. BMC Med Res Methodol 2004, 4:10.

24. Brisson M, Van d V, De WP, Boily MC: The potential cost-effectiveness of prophylactic human papillomavirus vaccines in Canada. Vaccine 2007, 25:5399-5408.

25. Kim JJ, Goldie SJ: Health and economic implications of HPV vaccination in the United States. N Engl J Med 2008, 359:821-832.

26. Kulasingam SL, Benard S, Barnabas RV, Largeron N, Myers ER: Adding a quadrivalent human papillomavirus vaccine to the UK cervical cancer screening programme: A cost-effectiveness analysis. Cost Eff Resour Alloc $2008,6: 4$.

27. Elbasha EH, Dasbach EJ, Insinga RP: Model for assessing human papillomavirus vaccination strategies. Emerg Infect Dis 2007, 13:28-41.

28. Sinnott PL, Joyce VR, Barnett PG: Preference Measurement in Economic Analysis VA Palo Alto, Health Economics Resource Center: Guidebook. Menlo Park CA; 2007.

\section{Pre-publication history}

The pre-publication history for this paper can be accessed here: http://www.biomedcentral.com/1471-2458/12/153/prepub

doi:10.1186/1471-2458-12-153

Cite this article as: Shi et al:: Impact of genital warts on health related quality of life in men and women in mainland China: a multicenter hospital-based cross-sectional study. BMC Public Health 2012 12:153.

\section{Submit your next manuscript to BioMed Central and take full advantage of:}

- Convenient online submission

- Thorough peer review

- No space constraints or color figure charges

- Immediate publication on acceptance

- Inclusion in PubMed, CAS, Scopus and Google Scholar

- Research which is freely available for redistribution

Submit your manuscript at www.biomedcentral.com/submit
Ciomed Central 